\title{
Implementation of eWAR system in the neutralization of selected chemicals in building ventilation systems
}

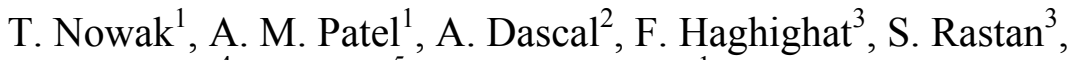 \\ E. Morofsky ${ }^{4}$, I. Butler ${ }^{5} \&$ J. A. Kozinski ${ }^{1}$ \\ ${ }^{1}$ Energy and Environmental Research Laboratory, McGill University, \\ Canada \\ ${ }^{2}$ Faculty of Medicine, McGill University, Canada \\ ${ }^{3}$ Department of Building, Civil and Environmental Engineering, \\ Concordia University, Canada \\ ${ }^{4}$ Public Works and Government Services, Canada \\ ${ }^{5}$ Department of Chemistry, McGill University, Canada
}

\begin{abstract}
This paper presents an Early Warning and Response System (eWAR) system capable of preventing casualties and mass hysteria in the event of a possible chemical release, be it criminal or accidental (in the case of an industrial spill). Experiments are conducted on a lab-scale set-up which uses a wireless computer interface to monitor important ventilation parameters such as air-velocity, humidity, pressure, temperature and their influence on in-duct levels of Volatile Organic Compounds (VOCs) and airborne contaminants. This system is designed to reduce the severity of various airborne contaminants, including some toxic agents, only when concentration of contaminants, regardless of type, generate some abnormal pulses as compared with the average day-to-day normal pulses of indoor and outdoor air. This capability of the system to be activated and deactivated avoids the additional energy and maintenance costs associated with continuous filtration, the frequent regeneration of filter media as well as the cost of additional air handling units to compensate for pressure drops caused by filters.

Preliminary results have been obtained using various chemicals and different types of activated carbons (as filters); they show that the in-duct concentration can in fact be controlled and significantly reduce the severity of some release scenarios.
\end{abstract}

Keywords: HVAC, activated carbon, building protection, early warning. 


\section{Introduction}

The mechanical Heating, Ventilation and Air Conditioning (HVAC) HVAC systems are the most efficient means of delivering clean air throughout a building. Unfortunately, in some cases, this air may contain unwanted or hazardous substances, for example, environmental pollutants and Toxic Chemical Agents (TCA). By delivering contaminated air, the HVAC can involuntarily act as a distribution platform for various kinds of contaminants including TCAs.

Two features make HVAC systems appealing targets for terrorists. First, when toxic airborne chemicals are injected into a ventilation system, the duct installation acts as a delivery system and distributes them throughout the building in a relatively short time period. The second potentially dangerous feature of the ventilation system is the fact that it has many supply duct outlets distributed evenly throughout the building. This allows a mixture of air and TCAs to be delivered directly to an area occupied by people.

\subsection{Chemical agents}

Toxic chemical agents can be released in the form of aerosol, gas or vapours. The two most likely forms of a TCA release into the building's ventilation system are gas injection and liquid agent spill. The main reason why these forms of agent delivery are so likely is the simplicity of the delivery equipment. For example, in the case of gas injection, a release setup can be as simple as a gas container equipped with a valve. In the case of a liquid agent, a simple spill within a duct system can lead to contamination of the entire building.

In contrast, an aerosol release would require a more complicated release setup most likely comprising an agent container, an aerosol generator and a power source. Such a method of delivery would be more complicated and therefore less practical.

\subsection{Approach}

In order to protect the building's ventilation system - and consequently the building's inhabitants - against TCAs, certain actions can be proposed.

The engineering approach to building protection is based on the removal of chemical agents from the air stream by continuous filtration (activated carbons, zeolites and silica gel), wet and dry scrubbers, and High Efficiency Particulate Air (HEPA) filtration [1-5]. These engineering-based solutions are used for both the removal of chemical agents from an incoming air stream as well as from the recirculated air stream.

However, any attempts to incorporate continuous filtration in the protection of the building against TCAs would create a significant increase in operating costs. This is primarily because of the higher energy demand caused by flow restrictions created by the filtration medium. In addition, maintenance costs and filter medium replacements would be added to the overall operational expenses. 
In order to overcome these disadvantages, an alternative approach combining the removal efficiency of continuous filtration with the benefits of noncontinuous filtration (energy conservation) needs to be implemented. Such an approach would provide a more energy-efficient system while still offering efficient protection against hazardous substances.

These objectives can be achieved by the development of a system capable of early event detection and in-duct neutralization of contaminants.

The eWAR system [1] combines non-specific event detection (Photo Ionization Detector, PID) with in-duct agent neutralization, thus preventing further migration of the intentionally released substances throughout the ventilation system. In addition, the eWAR system in its present form is designed to protect the building against chemical agents released in the form of liquid spills and gas injection. Therefore, this paper addresses only these forms of TCA release. However, in future work, special attention will be paid to the protection of buildings against TCAs delivered in aerosolized form.

\section{Experimental facility}

The eWAR installation [1] was modified for the purpose of this work in order to conduct release and filtration experiments and also to ensure the best system performance. The modified eWAR setup is shown in Figure 1.

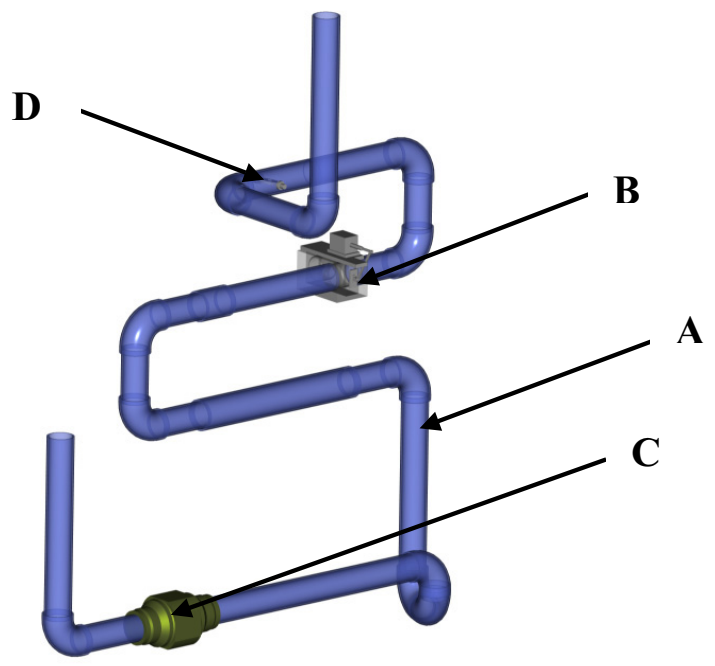

Figure 1: eWAR experimental facility.

The air duct system (A), shown in Figure 1, is made of transparent PVC pipes, with a diameter of $52 \mathrm{~mm}$, assembled to form an air supply duct. The total length of the eWAR duct is approximately $6900 \mathrm{~mm}$.

To minimize the effect of air by-passing the filter section (B), the eWAR filter, packed with either Granular Activated Carbon (GAC) or Activated Carbon Fibers (ACF), was placed into the filter assembly and sealed to ensure air- 
tightness. The system provides a control mechanism for non-continuous, ondemand operation of the filter. Activation of the filters is controlled by a computer system. The adsorbent bed of the filter is approximately $20 \mathrm{~mm}$ in depth and $52 \mathrm{~mm}$ in diameter.

Movement of the air medium through the eWAR installation was achieved using a lab-scale blower (C). The air velocity was adjusted by varying the blower speed and adjusting the position of the air damper (D). For this series of tests, the air velocity was set to $1.5 \mathrm{~m} / \mathrm{s}$.

The speed of the blower and the position of the air damper were controlled by the eWAR control system.

\subsection{Experimental methodology}

\subsubsection{The release methods}

As mentioned earlier, the experimental procedure proposed in this work considers a building's air supply system as a potential target of an intentional TCA release. Therefore, the release methods used during the tests simulate a situation where this part of the system is attacked.

The location of the intentional release impacts a number of factors that must be considered in the analysis of the agent's behaviour after release. In the case of the experiments described in this paper, the values of air humidity and temperature were considered to be constant because the air drawn into the eWAR installation was already pre-conditioned by the HVAC system of the laboratory where the tests were conducted. The humidity and temperature of the supplied air were measured by the eWAR control system. The typical values during the tests are shown in Table 1.

Table 1: $\quad$ eWAR experimental conditions.

\begin{tabular}{|c|c|c|}
\hline eWAR parameters & DMMP & Isobutylene \\
\hline Temperature & $21^{\circ} \mathrm{C}$ & $21^{\circ} \mathrm{C}$ \\
\hline Humidity & $30-31 \% \mathrm{RH}$ & $30-31 \% \mathrm{RH}$ \\
\hline Air velocity & $1.5 \mathrm{~m} / \mathrm{s}$ & $1.5 \mathrm{~m} / \mathrm{s}$ \\
\hline
\end{tabular}

\subsubsection{Test substances}

2.1.2.1 Isobutylene To simulate the release of a chemical agent in gaseous form, a mixture of air and isobutylene purchased from MEGS was used. The concentration of isobutylene in air was $200 \mathrm{ppm}$. The gas was injected into the eWAR experimental facility using a setup composed of a gas container, a pressure regulator, a flow meter and an injection nozzle. The isobutylene injection flow rate was set to $221 / \mathrm{min}$ in order to obtain an in-duct isobutylene concentration of $20 \mathrm{ppm}$. The distance between the injection point and the filter was set to $2260 \mathrm{~mm}$ and the distance between the system inlet and the injection point was $2190 \mathrm{~mm}$. 
2.1.2.2 Dimethyl Methylphosphonate (DMMP) To simulate a release of the liquid agent in the form of a spill, dimethyl methylphosphonate (DMMP) with purity $97 \%$ and purchased from Sigma Aldrich, Fluka was used. The liquid DMMP was introduced into the reservoir and formed a liquid pool having a surface area equal to $17 \mathrm{~cm}^{2}$. From the pool's surface, the agent's vapours evaporated into the air stream.

This release method and setup ensured a constant liquid level and pool surface area. Therefore, only the air velocity and the evaporative cooling process could influence the evaporation rate. It can be assumed that the agent's in-duct concentration was constant. The reservoir was located $730 \mathrm{~mm}$ from the system's inlet. The distance between the reservoir and the filter was set to $3720 \mathrm{~mm}$.

The DMMP was used to investigate its behaviour after release into the air stream as well as test the system response to a substance similar to Sarin. DMMP is an organophosphate and thus belongs to the same family as nerve agents and shows structural and physical similarities to Sarin (GB). This substance can be used to simulate the behaviour of GB [4] and [6] after its release into the ventilation system. Experiments conducted using DMMP also provided crucial information about the PID detection performance in terms of its measurement accuracy and its applicability as a continuous monitoring sensor for the eWAR system.

It should be mentioned that both delivery methods were considered to be continuously supplying a steady input of the agent into the eWAR experimental installation. Therefore, during the filtration experiments, the tested filters were exposed to a continuous stream of air containing constant and equal amounts of the agent over the duration of the test.

2.1.2.3 Adsorbents and in-duct neutralization of chemical agents To investigate the in-duct agent neutralization concept, two kinds of adsorbents were used. These adsorbents were: activated carbon fibers, ACF-1603-20 purchased from American Kynol Inc. and granular active carbon, 6-14 mesh 5685A purchased from Fisher Scientific. The specific surface areas of these two adsorbents were $2000 \mathrm{~m}^{2} / \mathrm{g}$ and $1200 \mathrm{~m}^{2} / \mathrm{g}$ for ACF and GAC, respectively.

Prior to each experiment, each adsorbent was weighed on a laboratory scale and stored in a clean container to avoid contamination by VOCs and exposure to humidity. The amount of adsorbent used during each experiment was $20 \mathrm{~g}$ of GAC and $1.9 \mathrm{~g}$ of ACF. Before each test, a new batch of absorbent was packed into the filter holder and placed inside the filter assembly of the eWAR system.

\subsubsection{TCA detection}

The detection of a chemical agent release is achieved using a ppbRAE Photoionization Detector (PID), Model PGM-7240, courtesy of RAE Systems INC. USA. This sensor was not only able to detect the release event but also to measure the concentration of the released substance.

The signals obtained from the PID (VOCs concentration readings) are transferred directly to the control software where they are stored in an Excel file for data acquisition and further analysis. 
The measurement accuracy of the PID sensor is within the range of $\pm 10 \%$ of the actual concentration of the measured substance according to information provided by RAE Systems. As a result, all measurements reported will be affected by a measurement error equal to $10 \%$.

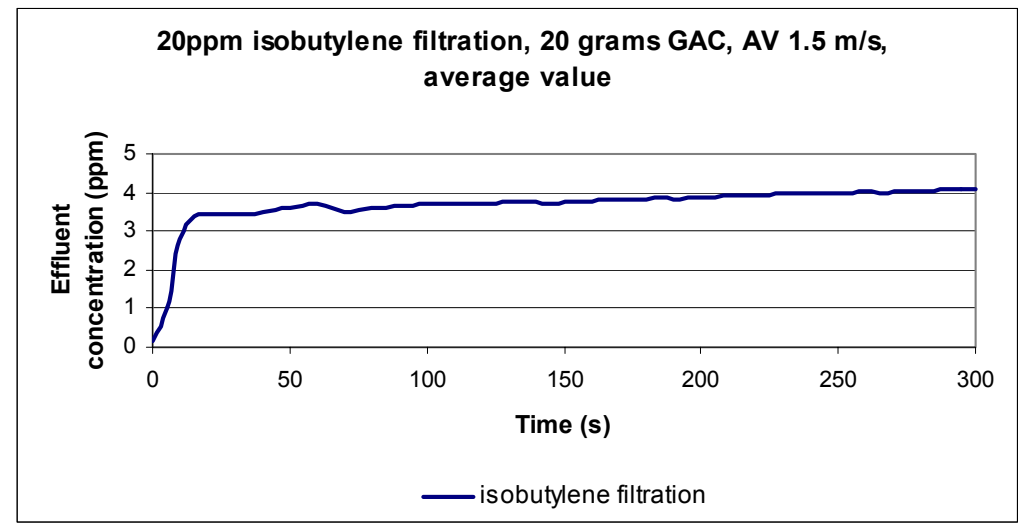

Figure 2: $\quad$ Isobutylene filtration by GAC filter.

\section{Results}

\subsection{Isobutylene filtration by GAC filter}

The curve shown in Figure 2 shows the average value of the isobutylene effluent concentration as a function of time. The filtration experiments were conducted for 300 seconds.

The average value was obtained based on the results of three separate experiments conducted under the same conditions (temperature, humidity and air velocity). Based on the results shown in Figure 2 it can be observed that the maximum influent isobutylene concentration equal to $20 \mathrm{ppm}$ was reached approximately $15 \mathrm{~s}$ after the release. This delay can be attributed to concentration build up time, detection and response time, and limitations of the isobutylene injection setup used during the tests. The minimum value of the effluent isobutylene concentration, corresponding to the $15^{\text {th }}$ second of the test, was approximately $3 \mathrm{ppm}$.

In addition, Figure 2 presents the dynamic response of the filtration medium when exposed to an air stream containing 20ppm of isobutylene. This particular curve was obtained for the filter packed with approximately $20 \mathrm{~g}$ of GAC. As can be seen, the concentration of isobutylene measured downstream from the GAC filter was within the range between $3 \mathrm{ppm}$ and $5 \mathrm{ppm}$. The removal efficiency described as the decrease of the effluent isobutylene concentration due to the filtration process - decreased from $85 \%$ at the beginning of the experiment to $75 \%$ at the end. Adsorption of the gas to the surface of the eWAR ducts is not corrected for.

The curve shown in Figure 3 presents the average value of the isobutylene effluent concentration as a function of time. This particular curve was obtained 
for the filter packed with $1.9 \mathrm{~g}$ of ACF. As with the GAC filtration experiments, there was a delay of $15 \mathrm{~s}$ between the moment of agent injection and the point when maximum in-duct concentration of was reached.

As can be seen in Figure 3, the concentration of isobutylene obtained downstream from the ACF filter was within the range between $5 \mathrm{ppm}$ and $11 \mathrm{ppm}$. Generally, the removal efficiency ranged between $75 \%$ at the beginning of the test and $45 \%$ at the end. Adsorption of the gas to the surface of the eWAR ducts is not corrected for.

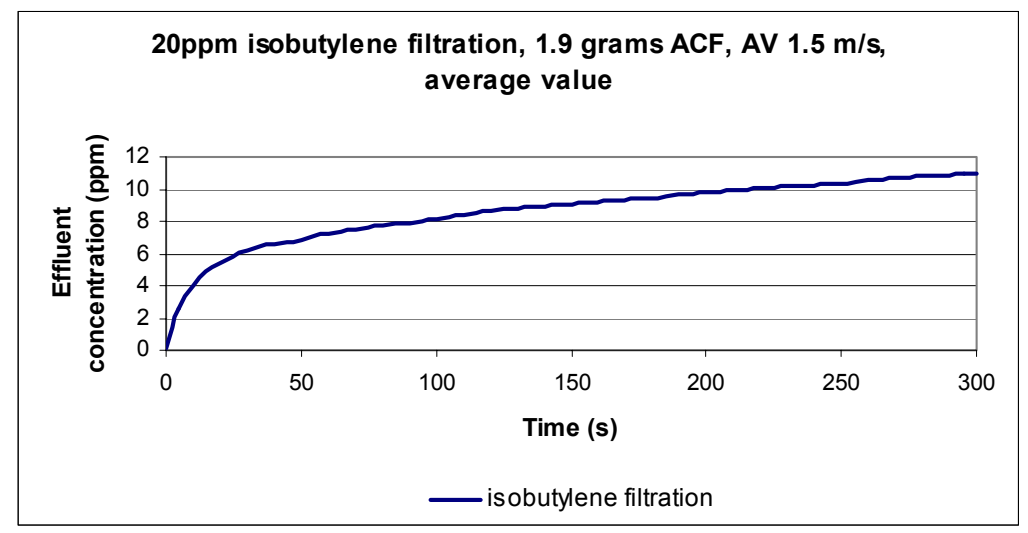

Figure 3: Isobutylene filtration by ACF filter.

\subsection{Isobutylene filtration by ACF filter}

If one compares Figures 2 and 3, it can be observed that in the case of ACF, the rate of increase of the isobutylene concentration is more pronounced. For example, the effluent concentration increased by $6 \mathrm{ppm}$ over $275 \mathrm{~s}$, whereas in the case of GAC, the effluent concentration increased only by $2 \mathrm{ppm}$, respectively. This situation can be explained by the fact that during the tests approximately 10 times more GAC (20g) was used than ACF (1.9g), allowing GAC to adsorp more isobutylene molecules due to a presence of more adsorption sites.

\subsection{DMMP filtration by GAC filter}

Figure 4 shows the average value of the DMMP effluent concentration as a function of time. The results were obtained with three independent experiments. The DMMP vapours were removed from the air stream using a filter packed with $20 \mathrm{~g}$ of GAC. There was a $5 \mathrm{~s}$ delay time between the introduction of the agent into the reservoir and the time at which the in-duct concentration reached the maximum value.

As can be seen in Figure 4 after approximately100s there is a decrease in the DMMP concentration. This situation is caused by contamination of the PID sensor, which in result leads to inaccurate readings of the agent concentration. Therefore, in this particular case, only the time period from the beginning of the test to approximately100s can be considered as meaningful. 
According to Figure 4, the effluent DMMP concentration was between $0.1 \mathrm{ppm}$ and $0.45 \mathrm{ppm}$. As the influent DMMP concentration was equal to $2.7 \mathrm{ppm}$, this gives a maximum removal efficiency of $96 \%$ at the beginning of the test and a minimum of $75 \%$ at the $100^{\text {th }}$ second of the test. Adsorption of the DMMP vapours to the surface of the eWAR ducts is not corrected for.

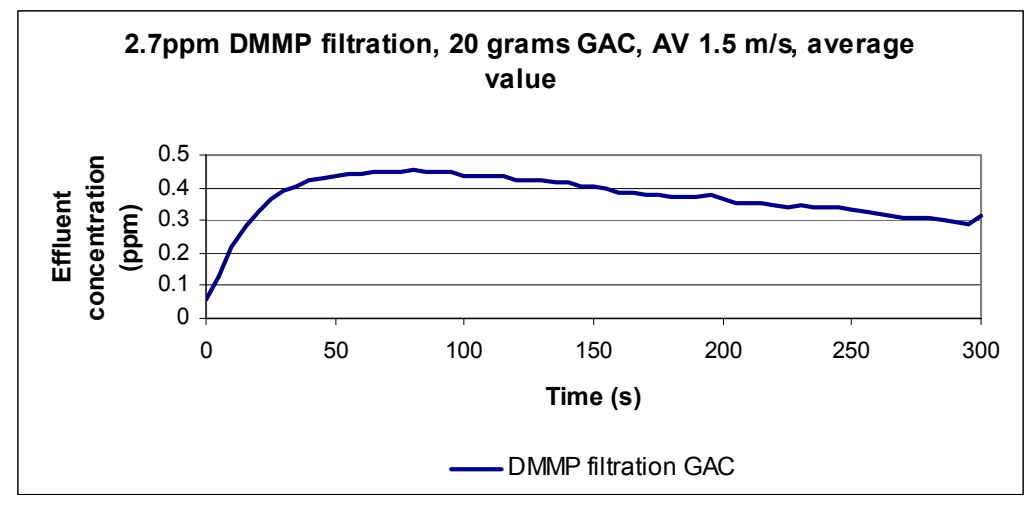

Figure 4: DMMP filtration by GAC filter.

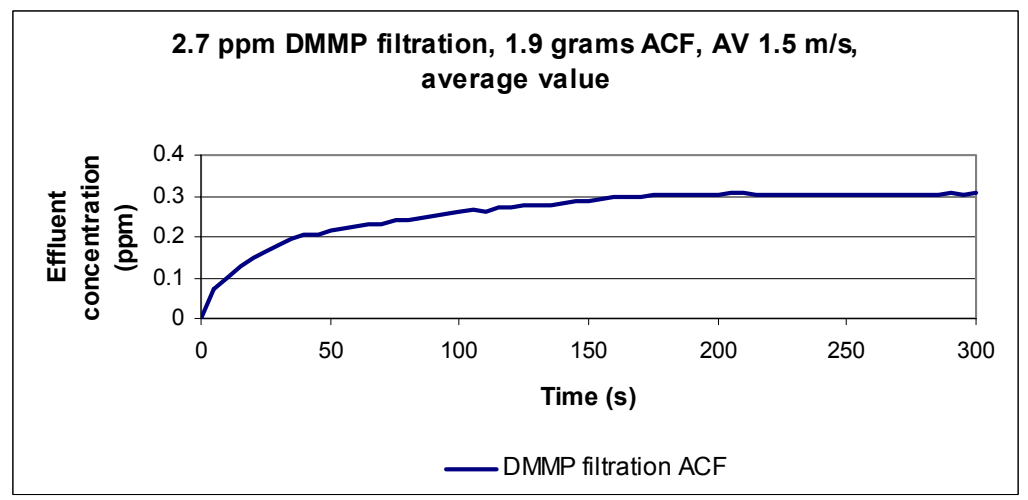

Figure 5: DMMP filtration by ACF filter.

\subsection{DMMP filtration by ACF filter}

Figure 5 shows the average effluent concentration of DMMP as a function of time. The average values were obtained based on three separate filtration experiments conducted using a filter packed with $1.9 \mathrm{~g}$ of ACF. The delay time between the introduction of the agent into the reservoir and the time at which the in-duct concentration reached its maximum value was approximately $5 \mathrm{~s}$.

After the initial increase of the DMMP effluent concentration until approximately the $150^{\text {th }}$ second of the test, its value stabilized at the level of $0.3 \mathrm{ppm}$ and remained stable for the remainder of the test. As the influent DMMP concentration was equal to $2.7 \mathrm{ppm}$, the obtained removal efficiency ranged from 
a maximum of $97 \%$ at the beginning of the experiment to $89 \%$ at the end. Adsorption of the DMMP vapours to the surface of the eWAR ducts is not corrected for.

Moreover, unlike as with GAC, no PID contamination by DMMP was observed during these experiments.

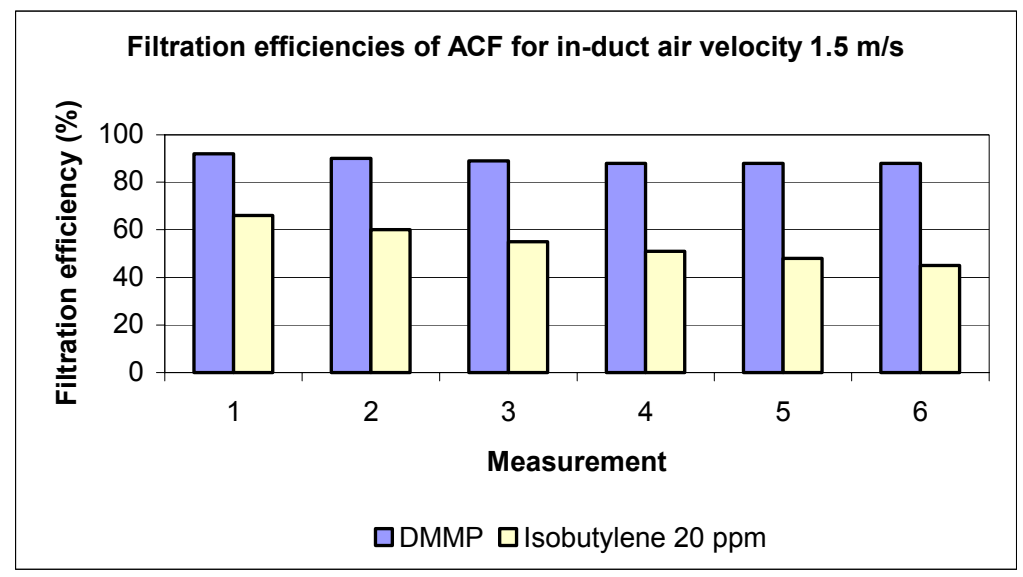

Figure 6: The removal efficiency of the ACF filter.

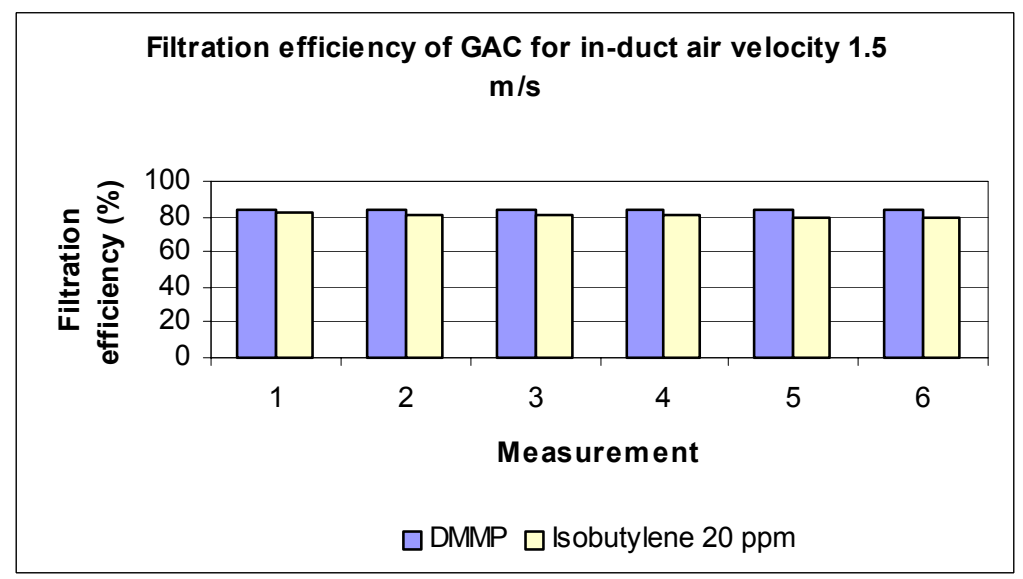

Figure 7: The removal efficiency of the GAC filter.

\section{Conclusions}

Figures 6 and 7 show the values of the removal efficiencies obtained for two different test chemicals and adsorbents. Each measurement point shown in Figures 6 and 7 represents the actual measurement taken at the $50^{\text {th }}$ second of each test and its corresponding value of removal efficiency.

In the case of DMMP filtration, because of contamination of the PID sensor, the values of the removal efficiency after the $100^{\text {th }}$ second can be considered 
only as approximate values. This assumption regarding the values of the removal efficiency for the GAC filter was made based on observation of the trend of stabilization of the effluent concentration, as illustrated in Figure 2.

On the basis of these figures, it can be concluded that the filtration performance of ACF strongly depends on the influent concentration of the test substance. As mentioned, this situation is most likely caused by the fact that a relatively small amount of the adsorbent was used.

From Figure 7, one may suggest that the filtration performance of GAC is nearly independent of the influent concentration of the test substance.

In the case of isobutylene filtration the reported removal efficiencies for GAC and $\mathrm{ACF}$ were from $85 \%$ to $75 \%$ and from $75 \%$ and $45 \%$, respectively.

In the case of the DMMP filtration experiments, the values of the removal efficiencies reported in this work were from $96 \%$ to $75 \%$ for the GAC filter and from $97 \%$ to $90 \%$ for the ACF filter. Despite the significantly smaller amount of $\mathrm{ACF}$, the removal efficiency was better than that obtained for GAC.

In addition, the concept of on-demand, just-in-time, in-duct neutralization could be achievable by using controlled and limited amounts of adsorbent for filtration. Moreover, depending on the influent concentration of the test substance and the type of adsorbent, the system can operate at a removal efficiency of at least 75\% (GAC-isobutylene), 45\% (ACF-isobutylene), 89\% (ACF- DMMP) for a period of 300s and 75\% (GAC-DMMP) for a period of $100 \mathrm{~s}$. As a result, the main goal of protecting the building's occupants against TCAs can be achieved by, at least, partially neutralizing the concentration of induct airborne contaminants and hazardous substances.

\section{References}

[1] Rastan, S, Nowak, T., Kozinski, J., Haghighat, F., Morofsky, E., Foo, S., Voinea, S. and Rous, J., An early warning and response system for buildings at risk to toxic airborne materials, Safety and Security Engineering, WIT Trans. on The Built Environment, 82, pp. 427-438, WIT Press, 2005

[2] Kowalski, W. J. and Bahnfleth, W. P., Immune-building technology and bioterrorism defence, HPAC Engineering, January, pp. 57-62, 2003.

[3] Kowalski, W. J., Bahnfleth, W. and Musser, A. Modeling immune buildings systems for bioterrorism defense, Journal of Architectural Engineering, June 2003.

[4] L. A. Jonas and J. A. Rehrmann, The Kinetic of Adsorption of OrganoPhosphorus Vapors from Air Mixtures by Activated Carbons, Carbon, Vol. 10, pp.657- 663, 1972

[5] Jonas, L.A. and Rehrmann, J.A., The kinetic of adsorption of organophosphorus vapors from air mixtures by activated carbons, Carbon, 10, pp.657-663, 1972.

[6] Sun, Y. and Ong, K. Y., Detection technologies for chemical warfare agents and toxic vapors, CRC Press, 2005 